TESTING STRICTLY CONCAVE RATIONALITY

by

Rosa L. Matzkin and Marcel K. Richter

Discusston Paper No. 239, July 1987

\author{
Center for Economic Research \\ Department of Economics \\ University of Minnesota \\ Minneapolis, Minn 55455
}




\title{
TESTING STRICTLY CONCAVE RATIONALITY
}

\author{
by \\ Rosa L. Matzkin \\ Cowles Foundation \\ Yale University \\ and \\ Marcel K. Richter \\ University of Minnesota
}

\begin{abstract}
We prove that the Strong Axiom of Revealed Preference tests the existence of a strictly quasiconcave (in fact, continuous, generically $C^{\infty}$, strictly concave, and strictly monotone) utility function generating finitely many demand observations.

This sharpens earlier results of Afriat, Diewert, and Varian that tested ("nonparametrically") the existence of a piecewise linear utility function that could only weakly generate those demand observations. When observed demand is also invertible, we show that the rationalizing can be done in a $C^{\infty}$ way, thus extending a result of Chiappori and Rochet from compact sets to all of $R^{n}$.

For finite data sets, one implication of our result is that even some weak types of rational behavior - maximization of pseudotransitive or semitransitive preferences - are observationally equivalent to maximization of continuous, strictly concave, and strictly monotone utility functions.
\end{abstract}

KEYWORDS: Rational choice, revealed preference, strictly concave utility, nonparametric tests. 


\title{
TESTING STRICTLY CONCAVE RATIONALITY*
}

by

\author{
Rosa L. Matzkin \\ and \\ Marcel K. Richter**
}

\section{INTRODUCTION}

In applied economics, consumers typically maximize continuous, strictly quasi-concave, and monotone utility functions. Even strict concavity is often assumed. What restrictions do these special assumptions put on observable data? Can we test demand behavior to see whether it maximizes such a special function?

For a finite number of observations, we will give a complete answer (Theorem 1): Houthakker's Strong Axiom of Revealed Preference is a necessary and sufficient behavioral test for such "special rationality." This will imply that the special rationality is observationally equivalent to the much weaker hypothesis that the demand function maximizes some reflexive, transitive, and total preference: No finite set of data can distinguish between those assumptions. In fact, our results imply that finite sets of data cannot distinguish even much weaker types of rationality from the special rationality (Theorem 3 ). Our proof is based on a result that provides a constructive method for obtaining continuous, generically $C^{\infty}$, strictly concave, strictly monotone utility-rationalizations (Theorem 2).

In Section 5 we relate our results to work by Afriat, Diewert, and Varian. That line of work - sometimes under the name of "nonparametric demand analysis" - tested for the existence of a piecewise linear utility, which could only rationalize demand in a much weaker sense. We also relate our work to the recent paper of Chiappori and Rochet on $C^{\infty}$ rationalizations.

In Section 6 we give some applications of our results.

*The main part of Theorem 1 of this paper was presented to the Midwest Mathematical Economics Conference, October 27, 1985.

**The assistance of the National Science Foundation, Grant SES-8510620, is gratefully acknowledged. 


\section{RATIONALTYY}

We study demand data for $n$ commodity types, where each bundle of commodities can be represented by a vector in some convex subset $X$ of $R^{n}$. (Commonly $X$ is assumed to be a subset of $R_{\underline{2}}^{n}$, but we do not require that.)

We are interested in competitive consumers, so we denote by $B(p, m)$ the budget set determined by price vector $p=\left(p_{1}, \ldots, p_{n}\right) \in R_{>}^{n}$ and income $m \in R_{\geqq}^{l}$ :

$$
B(p, m)=\{x \in X: p \cdot x \leqq m\} .
$$

Often we write $(p, m)$ for $B(p, m)$. We denote by $C$ the family of all such budget sets. Sometimes we are interested in a particular subfamily $B \subset \tilde{C}$. For example, in this paper, we study choices from a finite collection $\underline{B}$ of budgets.

The set of bundles chosen under budget $B(p, m)$ will be denoted by $h(p, m)$. It is reasonable to assume that $h(p, m) \subset B(p, m)$. This defines then a correspondence $h$, which we call a choice. Of course, in the classical case of a demand function, $h$ is singleton valued.

Using the notions of Richter (1971), we will call the choice $h$ rational (with respect to $(X, B)$ ), if there exists a binary relation $>$ on $X$ such that, for all $(p, m) \in \underset{\sim}{B}$,

$$
h(p, m)=\left\{x \in B(p, m): \forall y_{y \in B}(p, m) x>y\right\} .
$$

In other words, the set of chosen elements under budget $B(p, m)$ is exactly the set of preferred elements from $B(p, m)$. We say that $>$ is a rationalization for $h$, and that $\succ$ rationalizes $h$.

There are many subsidiary types of rationality: one can talk of transitive-rationality (for rationalization by a transitive relation $\gg$ ), or total-rationality (for rationalization by a total relation $\succsim$ ), etc. A very important type of rationality is regular-rationality, in which there is a reflexive, transitive, and total rationalization $\rightarrow$. And then, of course, one can consider utilityrationality, in which there is a rationalization that is representable by a numerical utility function $U$ on $X$ :

$$
h(p, m)=\left\{x \in B(p, m): \forall y_{y \in B(p, m)} U(x) \geqq U(y)\right\},
$$

for all $(p, m) \in B$. Even more demanding, of course, would be to seek a rationalization that has a continuous, strictly concave, and strictly monotone utility representation. For brevity, we will call this special-rationality.

We say that a choice is exhaustive (on $\underline{B}$ ) if it satisfies the budget equality

$$
p \cdot x=m
$$

for all $x \in h(p, m)$ and all $(p, m) \in B$. Of course, if only price vectors $p$ and commodity vectors $x$ are observed, but not incomes $m$, then for each such $p$ and $x$ we may define an $m=p \cdot x$, and then $h$ is automatically exhaustive. 
The main result of this paper can be viewed in three ways. First, it gives an empirical test for the existence of a continuous (and generically $C^{\infty}$ ), strictly concave, and strictly monotone utility-rationalization for any finite set of demand data (Theorem 1). Second, it gives a procedure for constructing such a rationalization (Theorem 2). Third, it shows that certain low types of rationality are actually equivalent to the much higher special-rationality type (Theorem 3 ).

\section{REGULAR AND SPECIAL RATIONALITY}

First we state some definitions. Following Richter $(1971,1979)$ we define the binary relation $S$ on $X$ by: for all $x, y \in X$,

$$
x S y \Leftrightarrow \exists B_{B_{\in} B} x \in h(B) \& x \neq y \in B
$$

Let $H$ be the transitive closure ${ }^{1}$ of $S$. Then Houthakker's Strong Axiom of Revealed Preference can be stated as:

$H$ is asymmetric.

It is known from Richter $(1966,1971)$ that, for demand functions, the Strong Axiom is equivalent to regular-rationality.

We say that a set $A \subset R^{n}$ is generic if it includes an open dense set whose complement is null (of Lebesgue measure zero). Equivalently, the complement of a generic set is small in the sense that it is a subset of a closed nowhere dense null set. We say that a property holds generically, if it holds on a generic set.

We will prove that a very high type of rationality follows from just the Strong Axiom.

Theorem 1. Let $h$ be an exhaustive demand function defined on a finite subset $B$ of $C$. Then $h$ has a special-rationalization $U$ if and only if $h$ satisfies the Strong Axiom of Revealed Preference. Furthermore, when such a $U$ exists, it can be chosen to be defined on all of $R^{n}$, and be generically $C^{\infty}$.

REMARK 1. There is no hope, however, of obtaining differentiability of rationalizations. In the two observations of Figure $1 \mathrm{a},{ }^{2}$ for example, we have $x=h\left(\bar{p}_{1}, \bar{p}_{2}, \bar{m}\right)=h\left(\hat{p}_{1}, \hat{p}_{2}, \hat{m}\right)$. If there were a differentiable utility rationalization, then Lagrange's theorem on constrained maximization would guarantee the existence of $\bar{\lambda}$ and $\hat{\lambda}$ satisfying $D_{i} u\left(x_{1}, x_{2}\right)=\bar{\lambda} \bar{p}_{i}=\hat{\lambda} \hat{p}_{i}$ for $i=1,2$. Both $\bar{\lambda}$ and $\hat{\lambda}$ must be zero; otherwise $D_{1} u\left(x_{1}, x_{2}\right) / D_{2} u\left(x_{1}, x_{2}\right)=\bar{p}_{1} / \bar{p}_{2}=\hat{p}_{1} / \hat{p}_{2}$, which

${ }^{1}$ The transitive closure of a relation $R$ is defined as the smallest transitive relation including $R$.

${ }^{2}$ The figure is essentially the same as in Chiappori and Rochet (1987). The interpretation is different, since they 
contradicts Figure 1a, since the budget lines have different slopes. So $D_{i} u\left(x_{1}, x_{2}\right)=0$ for $i=1,2$. Since $u$ is concave, $x$ globally maximizes $u$, contradicting the strict monotonicity of $u$.

Of course, without monotonicity, the differentiable strictly concave utility $u\left(x_{1}, x_{2}\right) \equiv\left(x_{1}-1\right)^{2}+\left(x_{2}-1\right)^{2}$ does rationalize Figure 1a. But even without requiring monotonicity, reasoning similar to the above shows that no differentiable strictly concave utility can rationalize the four observations of Figure $1 b$.

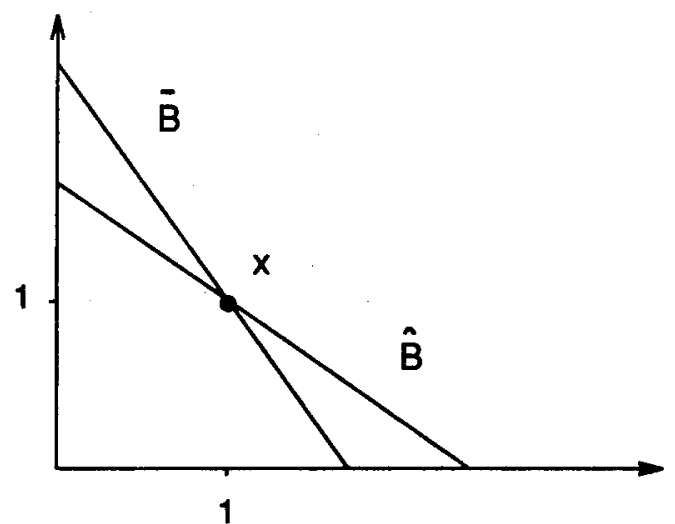

(a)

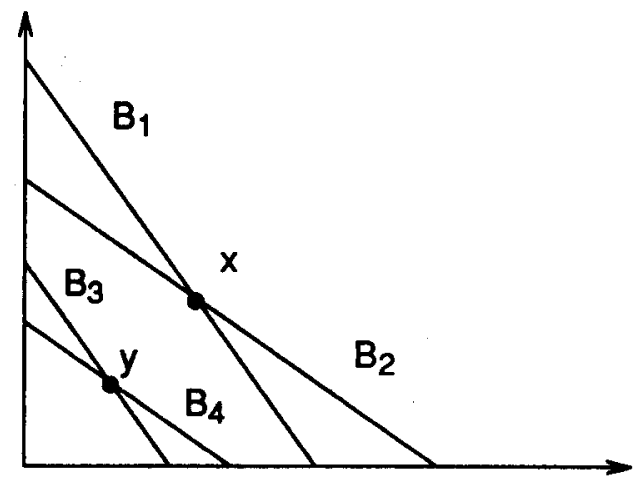

(b)

Figure 1

Nevertheless, despite Remark 1, we will show that a utility-rationalization can be found that is generically $C^{\infty}$.

REMARK 2. Theorem 1 gives an empirical test (the Strong Axiom) for determining whether a finite set of demand data can be rationalized by a continuous, strictly concave, and strictly monotone utility function. For it is clear that one can design algorithms to test, on any finite data set, whether the Strong Axiom holds. The next theorem also makes that clear, since it shows that satisfaction of the Strong Axiom is equivalent to solvability of a certain system of linear equalities and inequalities; and algorithms to test such solvability, and obtain solutions, are well known. ${ }^{3}$ In addition, it should be noted that the proof of Theorem 2 below will provide a constructive method for obtaining special-rationalizations from the system's solutions.

A proof of the main part of Theorem 1, for compact domains $X$, was given in Matzkin (1986). Here we present a very different proof, based on the fact that Theorem 1 is an immediate corollary of Theorem 2, which is proved in Section 4.

use a weaker notion of rationality (cf. Section 5 below).

${ }^{3} \mathrm{Cf}$. the discussion of Fourier elimination in Stoer and Witzgall (1970), Theorem 1.1.9 and Sections 1.2 and 1.3. 
Theorem 2. Let $h$ be an exhaustive demand correspondence on a finite subset $\underset{B}{B}=\left\{\left(p^{1}, m^{1}\right), \ldots,\left(p^{k}, m^{k}\right)\right\}$ of $\underset{\sim}{C}$. Let $x^{i} \in h\left(p^{i}, m^{i}\right)$ for $(i=1, \ldots, k)$. Then the following statements are equivalent:

a) $h$ satisfies the Strong Axiom of Revealed Preference.

b) There exists a continuous, strictly concave, and strictly monotone function $U$ rationalizing $h$ on $B$. (I.e., $h$ is special-rational.) (Optionally, $U$ can be chosen to be defined on all of $R^{\tilde{n}}$, and be generically $C^{\infty}$.)

c) There exist real numbers $u^{i}, u^{j}$ and $\lambda^{i}(i, j=1, \ldots, k)$ satisfying:

$$
\begin{array}{rlrl}
u^{i}+\lambda^{i} p^{i}\left[x^{j}-x^{i}\right] & >u^{j} & \text { for all } i, j=1, \ldots, k \text { with } x^{i} \neq x^{j} \\
\lambda^{i}>0 & \text { for all } i=1, \ldots, k \\
u^{i} & =u^{j} & \text { for all } i, j=1, \ldots, k \text { with } x^{i}=x^{j}
\end{array}
$$

(Optionally, the $\lambda^{i}$ can be chosen so that $\lambda^{i} p^{i} \neq \lambda^{j} p^{j}$ for $i \neq j$.)

d) $h$ is regular-rational.

REMARK 3. Conditions (c) are a strengthening ${ }^{4}$ of Afriat's inequalities (1967), p. 73 (Theorem). To prove that (c) implies (b) we follow his method, but modify his proof to obtain a stronger result. The modifications are necessary because our definition of rationality is stricter than his, and because we insist on strict concavity of the rationalizing utility. See Section 5 below for a more detailed comparison between his work and ours.

\section{PROOF OF THEOREM 2}

PROOF OF THEOREM 2. That (a) implies (c) is the assertion of Lemma 1 below. That (c) implies (b) is the assertion of Lemma 2 below. A fortiori, (b) (even without the parenthetical options) implies (d). That (d) implies (a) is known from Richter (1966).

Before reading Lemma 1 or its proof, the reader should note that Lemma $1^{\infty}$ (in the Appendix) and its proof constitute a much easier introduction. (The statement and proof of Lemma 1 are complicated by the fact that we can have data with $x^{i}=x^{j}$ even when $p^{i} \neq p^{j}$,

${ }^{4}$ Afriat used a weak inequality in his analogue of (3.3a). 
whereas the simpler Lemma $1^{\infty}$ applies only when the demand function $h$ is invertible.)

Lemma 1. Under the hypotheses of Theorem 2, if $h$ satisfies the Strong Axiom of Revealed Preference, then there exist $u^{i}, u^{j}, \lambda^{i}$ satisfying (3.3).

PROOF. We seek real numbers $u^{i}, u^{j}, \lambda^{i}(i, j=1, \ldots, k)$ that solve:

$$
\begin{aligned}
& u^{i}-u^{j}-\lambda^{i} p^{i}\left[x^{i}-x^{j}\right]>0 \quad \text { for all } i, j=1, \ldots, k \text { with } x^{i} \neq x^{j} \\
& \lambda^{i}>0 \quad \text { for all } i=1, \ldots, k \\
& u^{i}-u^{j}=0 \quad \text { for all } i, j=1, \ldots, k \text { with } x^{i}=x^{j},
\end{aligned}
$$

with $\lambda^{i} p^{i} \neq \lambda^{j} p^{j}$ for $i \neq j$. Defining $\alpha^{i j} \equiv p^{i}\left[x^{i}-x^{j}\right]$, we can rewrite this as:

$$
\begin{aligned}
u^{i}-u^{j}-\lambda^{i} \alpha^{i j}>0 & \text { for all } i, j=1, \ldots, k \text { with } x^{i} \neq x^{j} \\
\lambda^{i}>0 & \text { for all } i=1, \ldots, k \\
u^{i}-u^{j}=0 & \text { for all } i, j=1, \ldots, k \text { with } x^{i}=x^{j},
\end{aligned}
$$

with $\lambda^{i} p^{i} \neq \lambda^{j} p^{j}$ for $i \neq j$. Let $K$ be the number of pairs $(i, j)$ with $x^{i} \neq x^{j}$. Then we can rewrite this as:

$$
\begin{aligned}
& A r>0 \\
& C r=0,
\end{aligned}
$$

where $r=\left(u^{1}, \ldots, u^{k}, \lambda^{1}, \ldots, \lambda^{k}\right)$, and where $A$ and $C$ are matrices with $2 k$ columns, defined as follows. (An example of the matrices $A$ and $C$ follows in (4.4). It may be helpful to refer to that example while reading the definitions of $A$ and $C$.)

Matrix $A$ has $K+k$ rows. The first $K$ rows correspond to the left hand side of (4.2a), with 0 in all positions, except for a 1 in position $i$, and a -1 in position $j$, and $-\alpha^{i j}$ in position $k+i$; and the last $k$ rows correspond to the left hand side of (4.2b), with 0 in all positions, except for a 1 in position $k+i$.

Matrix $C$ corresponds to the left hand side of (4.2c). (If $C$ is not empty, there are obvious redundancies we can eliminate without changing the solutions of (4.3). For example, if $u^{i}-u^{j}=0$ is one of the lines of (4.2c), then we can eliminate the row corresponding to $u^{j}-u^{i}$.) Let $L$ be the number of rows in $C$.

As an example, the matrices $A$ and $C$ for just four observations, with $x^{1}, x^{2}$, and $x^{3}$ distinct, and $x^{3}=x^{4}$, looks look like this: 


$$
\begin{aligned}
& A=\left[\begin{array}{cccccccc}
1 & -1 & 0 & 0 & -\alpha^{12} & 0 & 0 & 0 \\
1 & 0 & -1 & 0 & -\alpha^{13} & 0 & 0 & 0 \\
1 & 0 & 0 & -1 & -\alpha^{14} & 0 & 0 & 0 \\
-1 & 1 & 0 & 0 & 0 & -\alpha^{21} & 0 & 0 \\
0 & 1 & -1 & 0 & 0 & -\alpha^{23} & 0 & 0 \\
0 & 1 & 0 & -1 & 0 & -\alpha^{24} & 0 & 0 \\
-1 & 0 & 1 & 0 & 0 & 0 & -\alpha^{31} & 0 \\
0 & -1 & 1 & 0 & 0 & 0 & -\alpha^{32} & 0 \\
-1 & 0 & 0 & 1 & 0 & 0 & 0 & -\alpha^{41} \\
0 & -1 & 0 & 1 & 0 & 0 & 0 & -\alpha^{42} \\
0 & 0 & 0 & 0 & 1 & 0 & 0 & 0 \\
0 & 0 & 0 & 0 & 0 & 1 & 0 & 0 \\
0 & 0 & 0 & 0 & 0 & 0 & 1 & 0 \\
0 & 0 & 0 & 0 & 0 & 0 & 0 & 1
\end{array}\right] \\
& C=\left[\begin{array}{cccccccc}
0 & 0 & 1 & -1 & 0 & 0 & 0 & 0 \\
0 & 0 & -1 & 1 & 0 & 0 & 0 & 0
\end{array}\right]
\end{aligned}
$$

To prove that a solution vector $r$ exists for (4.3), suppose not; we obtain a contradiction as follows. Since no such $r$ exists, then, by a Theorem of the Alternative ${ }^{5}$ there exists a $K+k$ dimensional vector $v$ and an $L$-dimensional vector $z$ such that: ${ }^{6}$

$$
\begin{aligned}
& v^{\prime} A+z^{\prime} C=(0, \ldots, 0) \\
& v \geq 0
\end{aligned}
$$

If some row $i$ of $C$ has a corresponding $z_{i}<0$, then we can, without changing the solutions of (4.3), replace that row by its negative, and replace $z_{i}$ in (4.5a) by $-z_{i}$. So in (4.5) we can without loss of generality assume that

$$
v \geq 0 \quad \& \quad z \geqq 0 .
$$

We will refer to the rows of $A$ according to their $\alpha$-terms: thus $\operatorname{Row}^{A}(i, j)$ is the row containing the term $-\alpha^{i j}$. If a $\operatorname{Row}^{A}(i, j)$ has a positive $v$-multiplier in (4.5), then we call $\operatorname{Row}^{A}(i, j)$ a weighted row. We will refer to the $i$-th column of matrix $A$ or $C$ as $\operatorname{Col}^{A}(i)$ or $\mathrm{Col}^{C}(i)$.

Define $\gamma_{i}$ terms by

\footnotetext{
${ }^{5}$ Cf. Stoer and Witzgall (1970), p. 10, Theorem (1.1.9).

${ }^{6} \mathrm{By} v \geq 0$ we mean $v \geqq 0 \& v \neq 0$.
} 


$$
z^{\prime} C=\left(\gamma_{1}, \ldots, \gamma_{k}, 0, \ldots, 0\right)
$$

Since $v \geq 0$, we can without loss of generality suppose $v_{1}>0$. Then (4.5) implies that we cannot have $-\alpha^{1 i}>0$ for all $i=1, \ldots, K\left(\right.$ since $\left.v^{\prime} \operatorname{Col}^{A}(k+1)=0\right)$. So without loss of generality we can assume that $-\alpha^{12} \leqq 0$, hence $x^{1} S x^{2}$. We will now show that there exists a weighted row $i \neq 1$ with $-\alpha^{2 i} \leqq 0$, hence

$$
x^{2} S x^{i}
$$

The second component, -1 , of the first row of $A$ clearly guarantees (by (4.5)) that either $i$ ) there is some weighted row of $A$ with 1 as its second component, or ii) $\gamma_{2}>0$. In case (i), say $\operatorname{Row}^{A}(2, i)$ is weighted and its second component is 1 . Then (4.5) clearly implies that we cannot have $-\alpha^{2 j}>0$ for all $j$, since $v^{\prime} \mathrm{Col}^{A}(k+2)=0$. So there is some $j$ with $-\alpha^{2 j} \leqq 0$, hence $x^{2} S x^{j}$, so (4.7) holds in case (i). In case (ii), we have $\gamma_{2}>0$. Then we will prove below

$$
\text { There exists some } j=1, \ldots, k \text { such that } \gamma_{j}<0 \& x^{j}=x^{2} \text {. }
$$

It follows then from (4.5) that there is some weighted $\operatorname{Row}^{A}(j, i)$ of $A$ with 1 in $\operatorname{Col}^{A}(j)$. Again by (4.5), it cannot be that all such rows $\operatorname{Row}(j, i)$ have $-\alpha^{j i}>0$. So we can choose an $i$ such that $-\alpha^{j i} \leqq 0$, hence $x^{j} S x^{i}$. Since $x^{j}=x^{2}$ by (4.8), this proves (4.7).

Continuing in this fashion by finite induction, we obtain $x^{1} S x^{2} S x^{3} S \cdots$. Since there are only finitely many columns in the matrix $A$, this forces a contradiction of the Strong Axiom, which prevents any "cycling" back to previous $x^{i}$. And this contradiction completes the proof of Lemma 1, subject to verification of (4.8).

To prove (4.8), let $J$ be the set of $j$ with $x^{j}=x^{2}$. Then if (4.8) were not true, we would have

$$
z^{\prime} \mathrm{Col}^{C}(j) \geqq 0 \text { for every } j \in J \text {. }
$$

By our hypothesis (ii),

$$
z^{\prime} \mathrm{Col}^{C}(2)=\gamma_{2}>0 .
$$

So (4.9) and (4.10) imply

$$
\begin{aligned}
0 & <\sum_{j \in J} \gamma_{j} \\
& =\sum_{j \in J} z^{\prime} \operatorname{Col}^{C}(j) \\
& =\sum_{j \in J} \sum_{i=1}^{L} z_{i}\left(\operatorname{Col}^{C}(j)\right)_{i} \\
& =\sum_{i=1}^{L} z_{i} \sum_{j \in J}\left(\operatorname{Col}^{C}(j)\right)_{i}
\end{aligned}
$$


$=0$.

The justification for (4.11e) is that, for each $i, \sum_{j \in J}\left(\operatorname{Col}^{C}(j)\right)_{i}=0$, because in each row $i$ of $C$ there are only two columns $j \in J$ with nonzero entries: one 1 and one -1 . The contradiction (4.11) proves (4.8).

Finally, because the inequalities $(4.2 \mathrm{a}, \mathrm{b})$ are strict, the $\lambda$ 's can clearly be chosen so that $\lambda^{i} p^{i} \neq \lambda^{j} p^{j}$ for $i \neq j$

REMARK 4. Instead of basing our proof of Lemma 1 on a Theorem of the Alternative, a proof could be obtained through an algorithm very much in the spirit of Varian's algorithm (1982).

Lemma 2. Under the hypotheses of Theorem 2, if there exist $u^{i}, u^{j}, \lambda^{i}$ satisfying (3.3), including the optional part, then $h$ has a special rationalization $U$, defined on all of $R^{n}$, and generically $C^{\infty}$.

PROOF. Part A: Definition of $U$. Let $u^{i}$ and $\lambda^{i}(i=1, \ldots, k)$ satisfy the inequalities (3.3). Since there are only finitely many inequalities, clearly there exists an $\varepsilon_{0}>0$ such that:

$$
\begin{aligned}
u^{i}+\lambda^{i} p^{i}\left[x^{j}-x^{i}\right]-\varepsilon_{0}>u^{j} & \text { for all } i, j=1, \ldots, k \text { with } x^{i} \neq x^{j} \\
\lambda^{i}>0 & \text { for all } i=1, \ldots, k \\
u^{i}=u^{j} & \text { for all } i, j=1, \ldots, k \text { with } x^{i}=x^{j} .
\end{aligned}
$$

Now let $T>0$, and define $g: R^{n} \rightarrow R^{1}$ by: ${ }^{7}$

$$
g\left(x_{1}, \ldots, x_{n}\right)=\left(x_{1}^{2}+\cdots+x_{n}^{2}+T\right)^{1 / 2}-T^{1 / 2} .
$$

Then

$$
\begin{aligned}
& g(x)>0 \Leftrightarrow x \neq 0 \\
& g(x)=0 \Leftrightarrow x=0 \\
& {\left[\frac{\partial g}{\partial x_{i}}(x)\right]<1 \text { for all } x \text { and } i=1, \ldots, n}
\end{aligned}
$$

$$
g \text { is strictly convex }
$$

${ }^{7}$ Here the superscripts are exponents, not indices. 
$g$ is differentiable.

Then by (4.12) we can pick $\varepsilon>0$ so small that

$$
\begin{aligned}
u^{i}+\lambda^{i} p^{i}\left[x^{j}-x^{i}\right]-\varepsilon g\left(x^{j}-x^{i}\right) & >u^{j} \text { for all } i, j=1, \ldots, k \text { with } x^{i} \neq x^{j} \\
\lambda^{i} & >0 \text { for all } i=1, \ldots, k \\
u^{i} & =u^{j} \text { for all } i, j=1, \ldots, k \text { with } x^{i}=x^{j} .
\end{aligned}
$$

Now, for each $i=1, \ldots, k$, define $\phi_{i}: R^{n} \rightarrow R^{1}$ by

$$
\phi_{i}(x) \equiv u^{i}+\lambda^{i} p^{i}\left[x-x^{i}\right]-\varepsilon g\left(x-x^{i}\right)
$$

Since $g$ is strictly convex, each $\phi_{i}$ is strictly concave. And clearly

$$
\phi_{i}\left(x^{i}\right)=u^{i} \quad(i=1, \ldots, k) .
$$

Now define $U: R^{n} \rightarrow R^{1}$ by

$$
U(x) \equiv \min \left\{\phi_{i}(x): i=1, \ldots, k\right\}
$$

for all $x \in X$. As the minimum of finitely many strictly concave functions, $U$ is strictly concave.

Part B: Monotonicity of $U$. We will choose $\varepsilon$ to guarantee strict monotonicity. Since (4.18) defines $U$ as the minimum of finitely many $\phi_{i}$ functions, it clearly suffices to show that each $\phi_{i}$ has everywhere a strictly positive partial derivative. From (4.16), the partial derivatives are given by

$$
\begin{aligned}
D_{j} \phi_{i}(x) & =\lambda^{i} p_{j}^{i}-\varepsilon D_{j} g\left(x-x^{i}\right) \quad \text { for all } j=1, \ldots, n \\
& >\lambda^{i} p_{j}^{i}-\varepsilon 1 \quad(\text { by }(4.14 c)) .
\end{aligned}
$$

Since there are only finitely many indexes $i=1, \ldots, k$, we can pick $\varepsilon$ so small that this is positive for all $i=1, \ldots, k$ and all $j=1, \ldots, n$.

Part C: $U$ rationalizes. As a first step in proving that $U$ rationalizes $h$, we will show that

$$
U\left(x^{j}\right) \geqq u^{j} \quad \text { for all } j=1, \ldots, k .
$$

If (4.20) were not true, then we would have

$$
\begin{aligned}
u^{j} & >U\left(x^{j}\right) \\
& =\phi_{i}\left(x^{j}\right) \quad \text { for some } i=1, \ldots, k \text { (by }(4.18) \\
& =u^{i}+\lambda^{i} p^{i}\left[x^{j}-x^{i}\right]-\varepsilon g\left(x^{j}-x^{i}\right) \quad(\text { by }(4.16))
\end{aligned}
$$

which contradicts inequality (4.15a) if $x^{i} \neq x^{j}$, and contradicts (4.15c) if $x^{i}=x^{j}$. So (4.20) holds. 
(Although it is not needed in our proof, we note here that, in fact, equality holds in (4.20). For

$$
\begin{aligned}
\exists i_{i=1, \ldots, k} U\left(x^{j}\right) & =\phi_{i}\left(x^{j}\right) \quad(\text { by }(4.18)) \\
& \leqq \phi_{j}\left(x^{j}\right) \quad(\text { by }(4.18)) \\
& =u^{j} \quad(\text { by }(4.17)) .
\end{aligned}
$$

The equality then follows from (4.20) and (4.22).)

Next we show that $U$ utility-rationalizes $h$. It is clearly sufficient to show that, for each $i=1, \ldots, k$,

$$
\forall y_{p^{i} y \leq m^{i}} y \neq x^{i} \Rightarrow U\left(x^{i}\right)>U(y) .
$$

Now for such $y$,

$$
\begin{aligned}
U(y) & =\min \left\{\phi_{j}(y): j=1, \ldots, k\right\} \quad(\text { by }(4.18)) \\
& =\min \left\{u^{j}+\lambda^{j} p^{j}\left[y-x^{j}\right]-\varepsilon g\left(y-x^{j}\right): j=1, \ldots, k\right\} \quad(\text { by }(4.16)) \\
& \leqq u^{i}+\lambda^{i} p^{i}\left[y-x^{i}\right]-\varepsilon g\left(y-x^{i}\right) \\
& <u^{i} \quad \text { since } y \neq x^{i} \quad(\text { by }(4.15 \mathrm{~b}), \text { the budget equality, and (4.14a)) } \\
& \leqq U\left(x^{i}\right) \quad(\text { by }(4.20)) .
\end{aligned}
$$

So (4.23) holds, and $U$ utility-rationalizes $h$.

Part D: Genericity of infinite differentiability. Define

$$
E \equiv\left\{x \in R^{n}: U \text { is not } C^{\infty} \text { at } x\right\} .
$$

Clearly

$$
E \subset \cup\left\{E_{i j}: i, j=1, \ldots, k \& i \neq j\right\}
$$

where

$$
E_{i j} \equiv\left\{x \in R^{n}: \phi_{i}(x)=\phi_{j}(x)\right\}
$$

Define

$$
f_{i j} \equiv \phi_{i}-\phi_{j}
$$

so

$$
E_{i j}=f_{i j}^{-1}(0)
$$


Now it is easily checked that for all small enough $\varepsilon>0$ (cf. $(4.15,16)$ ) we have, for all $x \in R^{n}$ and $i \neq j$,

$$
D f_{i j}(x) \neq(0, \ldots, 0)
$$

So it follows from the Implicit Function Theorem ${ }^{8}$ that

$$
f_{i j}{ }^{-1}(0) \text { is an }(n-1) \text {-dimensional } C^{\infty} \text { submanifold of } R^{n},
$$

hence $E_{i j}$ is the complement of an open dense subset of $R^{n}$. Then $E$, as a subset of a finite union of such sets, is the complement of a generic set.

\section{COMPARISON WITH OTHER RESULTS}

To put our results in context, we first mention two other notions of rationality. If we replace the " $=$ " in (2.3) by " $c$ " or " $\supset$ " we obtain two definitions of semirationality which we call subsemirationality and suprasemirationality, respectively. They are clearly much weaker concepts than the rationality notion of (2.3). Note, for example, that every constant function is a subsemirationalization of any demand correspondence.

We can use these definitions to clarify two main lines of research. Although the terminology may have been different, the revealed preference work of Samuelson (1938a, 1938b), Houthakker (1950), Uzawa (1971), and Richter (1966) has worked primarily with the stricter notion of rationality. On the other hand, a line of work by Afriat $(1967,1973)$, Diewert (1973), and Varian (1982) has used the weaker notion of subsemirationality.

Afriat (1967) stated several conditions on finite sets of demand data from which he proved subsemirationality ("utility consistency" or "utility hypothesis" in his terminology). Afriat showed that his consistency conditions were also equivalent to "normal utility consistency," which in our terminology would mean concave-utility-subsemirationality. Since only weak concavity was required, any constant function would again be such a subsemirationalization, ${ }^{9}$

Afriat was interested in not just proving existence of a utility subsemirationalization, but also in providing a method for actually calculating such a function. The particular method he used was further developed by Diewert, who obtained such a function by solving a linear programming problem. Varian restated Afriat's result and construction in terms of a Generalized Axiom of Revealed Preference, which was weaker than the Strong Axiom; and he gave an algarithm to find a solution for the $u^{i}, \lambda^{i}$ that, when the Generalized Axiom holds, satisfies Afriat's inequalities .

\footnotetext{
${ }^{8}$ Cf. Guillemin and Pollack (1974), p. 21, Preimage Theorem; Kahn (1980), p. 69, Proposition 3.1.

${ }^{9}$ And would be a counterexample to the claim (Afriat (1967), pp. 69, 74 (Corollary)) that cyclical consistency is a necessary consequence of utility consistency.
} 
Our conclusions are much sharper than these subsemirationality results. First, they provide rationality in the full sense of (2.3). Second, they also guarantee strict concavity and strict monotonicity of the utility-rationalization. (The earlier constructions were never strictly concave.) Like those earlier results, they are algorithmically testable, and our proof also shows how to actually construct a utility-rationalization.

Recently Chiappori and Rochet (1987) have strengthened the Strong Axiom hypothesis by adding what amounts to invertibility of the observed demand function. Then they showed that one can obtain, on any compact subset of $R_{\underline{z}}^{n}$, a $C^{\infty}$, monotone, subsemi-utility-rationalization. Although their formal definition of rationality is only subsemi-rationality, their strict concavity conclusion actually yields what we have called rationality.

After their results were published, we realized that the methods of our Theorem 2 could be used to strengthen their conclusion. As we show in Theorems $1^{\infty}$ and $2^{\infty}$, one can obtain a $C^{\infty}$ utility-rationalization on all of $R^{n}$.

Given a demand function $h: B \rightarrow X$, we say that $h$ is (homogeneously) invertible if, for all $(p, m),\left(p^{\prime}, m^{\prime}\right) \in B$ : if $(p, m)$ is not a positive scalar multiple of $\left(p^{\prime}, m^{\prime}\right)$ then $h(p, m) \neq h\left(p^{\prime}, m^{\prime}\right)$.

Theorem $1^{\infty}$. Let $h$ be an exhaustive demand function defined on a finite subset $B$ of $C$. Then $h$ has a $C^{\infty}$ special rationalization defined on all of $R^{n}$ if and only if $h$ satisfies the Strong Axiom of Revealed Preference and is invertible.

Theorem $1^{\infty}$ is an immediate corollary of

Theorem $2^{\infty}$. Let $h$ be an exhaustive demand correspondence on a finite subset $B=\left\{\left(p^{1}, m^{1}\right), \ldots,\left(p^{k}, m^{k}\right)\right\}$ of $C$. Let $x^{i} \in h\left(p^{i}, m^{i}\right)$ for $(i=1, \ldots, k)$. Then the following statements are equivalent:

a) $h$ satisfies the Strong Axiom of Revealed Preference and is invertible.

b) There exists a $C^{\infty}$, strictly concave, and strictly monotone function $U$ rationalizing $h$ on $B$. (I.e., $h$ is special-rational.) And $U$ can be defined on all of $R^{n}$.

c) There exist real numbers $u^{i}, u^{j}$ and $\lambda^{i}(i, j=1, \ldots, k)$ satisfying:

$$
\begin{aligned}
u^{i}+\lambda^{i} p^{i}\left[x^{j}-x^{i}\right]>u^{j} & \text { for all } i, j=1, \ldots, k \text { with } x^{i} \neq x^{j} \\
\lambda^{i}>0 & \text { for all } i=1, \ldots, k
\end{aligned}
$$

d) $h$ is regular-rational and invertible.

The proof of Theorem $2^{\infty}$ is given in the Appendix. It is basically a combination of a greatly simplified version of our proof of Theorem 2, and Chiappori and Rochet's convolution. 


\section{APPLICATIONS}

Our result has many applications. We mention several.

i) As already noted, Theorems 1 and 2 give necessary and sufficient conditions in empirically testable forms, for the existence of special utility-rationalizations on finite data sets. And Theorem 2 shows one way to construct such utilities.

Similar statements apply to $C^{\infty}$ special utility-rationalizations and Theorems $1^{\infty}$ and $2^{\infty}$.

ii) Given a finite number of budget-demand observations, can we pass through them a continuous demand function that comes from a utility-rational consumer? Theorem 1 shows that this is possible if and only if the observations satisfy the Strong Axiom. In particular, the Strong Axiom is well known to be necessary for regular-rationality. And if the Strong Axiom holds, then Theorem 1 shows that the demand can be utility-rationalized by a continuous (strictly monotone, strictly concave) function, which by standard results must generate a continuous demand function. (This is a continuous analogue of the $C^{\infty}$ version for compact sets, given in the Corollary of Chiappori and Rochet (1987).)

iii) Theorem 1 also allows us to sharpen Mas-Colell's approximation result for continuous demand functions. He showed that income Lipschitzian demands satisfying a certain boundary condition can be rationalized by a unique continuous preference; and furthermore, this preference can be approximated by monotone, concave, subsemirationalizations on finite subsets of demand data (Mas-Colell (1978), remarks preceding Theorem 4). Now we can replace his application of Afriat's result by an application of our Theorem 1; this strengthens his result so that the approximating preferences are full rationalizations, and they have utility functions that are strictly concave and strictly monotone.

iv) If we combine Theorem 1 with Kim's recent result (1987), that the Strong Axiom is equivalent to semitransitive-rationality (and to pseudotransitive-rationality), then we immediately obtain

Theorem 3. Let $h$ be an exhaustive demand function defined on a finite subset $B$ of $C$. Then $h$ is special-rational if and only if $h$ is semitransitive-rational (equivalently, pseudotransitive-rational).

For finite sets of data, then, empirical tests cannot distinguish between certain weak types of nontransitive rationality and the much stronger special-rationality. 


\section{APPENDIX}

PROOF OF THEOREM $2^{\infty}$. That (a) implies (c) is the assertion of Lemma $1^{\infty}$ below. That (c) implies (b) is the assertion of Lemma $2^{\infty}$ below. By Remark 1, (b) implies (d). That (d) implies (a) is known from Richter (1966).

Lemma $1^{\infty}$. Under the hypotheses of Theorem $2^{\infty}$ (Section 5 ), if $h$ satisfies the Strong Axiom of Revealed Preference and is invertible, then there exist $u^{i}, u^{j}, \lambda^{i}$ satisfying (5.1).

PROOF. Without loss of generality, we can suppose that the $\left(p^{i}, x^{i}\right)$ are distinct. Since $h$ is invertible, we can further assume that the $x^{i}$ are distinct. Then we seek real numbers $u^{i}, u^{j}, \lambda^{i}$ $(i, j=1, \ldots, k)$ that solve:

$$
\begin{aligned}
u^{i}-u^{j}-\lambda^{i} p^{i}\left[x^{i}-x^{j}\right] & >0 \quad \text { for all distinct } i, j=1, \ldots, k \\
\lambda^{i} & >0 \quad \text { for all } i=1, \ldots, k
\end{aligned}
$$

Defining $\alpha^{i j} \equiv p^{i}\left[x^{i}-x^{j}\right]$, we can rewrite this as:

$$
\begin{aligned}
u^{i}-u^{j}-\lambda^{i} \alpha^{i j}>0 & \text { for all distinct } i, j=1, \ldots, k \\
\lambda^{i}>0 & \text { for all } i=1, \ldots, k
\end{aligned}
$$

or:

$$
A r>0 \text {, }
$$

where $r \equiv\left(u^{1}, \ldots, u^{k}, \lambda^{1}, \ldots, \lambda^{k}\right)$, and where $A$ is a $k^{2}$ by $2 k$ matrix, defined as follows. (An example of the matrix $A$ follows in (7.4).)

The first $k(k-1)$ rows of $A$ correspond to the left hand side of (7.2a), with 0 in all positions, except for a 1 in position $i$, a -1 in position $j$, and $-\alpha^{i j}$ in position $k+i$; and the last $k$ rows correspond to the left hand side of (7.2b), with 0 in all positions, except for a 1 in position $k+i$.

As an example, the matrix $A$ for just four distinct observations looks look like this: 


$$
A=\left[\begin{array}{cccccccc}
1 & -1 & 0 & 0 & -\alpha^{12} & 0 & 0 & 0 \\
1 & 0 & -1 & 0 & -\alpha^{13} & 0 & 0 & 0 \\
1 & 0 & 0 & -1 & -\alpha^{14} & 0 & 0 & 0 \\
-1 & 1 & 0 & 0 & 0 & -\alpha^{21} & 0 & 0 \\
0 & 1 & -1 & 0 & 0 & -\alpha^{23} & 0 & 0 \\
0 & 1 & 0 & -1 & 0 & -\alpha^{24} & 0 & 0 \\
-1 & 0 & 1 & 0 & 0 & 0 & -\alpha^{31} & 0 \\
0 & -1 & 1 & 0 & 0 & 0 & -\alpha^{32} & 0 \\
0 & 0 & 1 & -1 & 0 & 0 & -\alpha^{34} & 0 \\
-1 & 0 & 0 & 1 & 0 & 0 & 0 & -\alpha^{41} \\
0 & -1 & 0 & 1 & 0 & 0 & 0 & -\alpha^{42} \\
0 & 0 & -1 & 1 & 0 & 0 & 0 & -\alpha^{43} \\
0 & 0 & 0 & 0 & 1 & 0 & 0 & 0 \\
0 & 0 & 0 & 0 & 0 & 1 & 0 & 0 \\
0 & 0 & 0 & 0 & 0 & 0 & 1 & 0 \\
0 & 0 & 0 & 0 & 0 & 0 & 0 & 1
\end{array}\right]
$$

To prove that a solution vector $r$ exists for (7.3), suppose not; we obtain a contradiction as follows. Since no such $r$ exists, then, by a Theorem of the Alternative ${ }^{10}$ there exists a $k^{2}$. dimensional vector $v$ such that ${ }^{11}$

$$
\begin{aligned}
& v^{\prime} A=(0, \ldots, 0) \\
& v \geq 0 .
\end{aligned}
$$

We will refer to the rows of $A$ according to their $\alpha$-terms: thus $\operatorname{Row}^{A}(i, j)$ is the row containing the term $-\alpha^{i j}$. If a $\operatorname{Row}^{A}(i, j)$ has a positive $v$-multiplier in (7.5), then we call $\operatorname{Row}^{A}(i, j)$ a weighted row. We will refer to the $i$-th column of matrix $A$ as $\operatorname{Col}^{A}(i)$.

Since $v \geq 0$, we can without loss of generality suppose $v_{1}>0$. Then (7.5) implies that we cannot have $-\alpha^{1 i}>0$ for all $i=1, \ldots, k\left(\right.$ since $\left.\nu^{\prime} \operatorname{Col}^{A}(k+1)=0\right)$. So without loss of generality we can assume that $-\alpha^{12} \leqq 0$, hence $x^{1} S x^{2}$. We will now show that there exists a weighted row $i \neq 1$ with $-\alpha^{2 i} \leqq 0$, hence

$$
x^{2} S x^{i}
$$

The second component, -1 , of the first row of $A$ clearly guarantees (by (7.5)) that there is some weighted row of $A$ with 1 as its second component. Say $\operatorname{Row}^{A}(2, i)$ is weighted and its second component is 1 . Then (7.5) clearly implies that we cannot have $-\alpha^{2 j}>0$ for all $j$, since

\footnotetext{
${ }^{10}$ Cf. Stoer and Witzgall (1970), p.10, Theorem (1.1.9); Gale (1960), p. 48, Theorem 2.9.

${ }^{11}$ By $v \geq 0$ we mean $v \geqq 0$ \& $v \neq 0$.
} 
$v^{\prime} \operatorname{Col}^{A}(k+2)=0$. So there is some $j$ with $-\alpha^{2 j} \leqq 0$, hence $x^{2} S x^{j}$, so (7.6) holds.

Continuing in this fashion by finite induction, we obtain $x^{1} S x^{2} S x^{3} S \cdots$. Since there are only finitely many columns in the matrix $A$, this forces a contradiction of the Strong Axiom, which prevents any "cycling"' back to previous $x^{i}$. And this contradiction completes the proof of Lemma $1^{\infty}$.

Lemma $2^{\infty}$. Under the hypotheses of Theorem $2^{\infty}$ (Section 5), if there exist $u^{i}, u^{j}, \lambda^{i}$ satisfying (5.1), then $h$ has a $C^{\infty}$-special-rationalization $U$ defined on all of $R^{n}$.

PROOF. First we follow Parts A and B of the proof of Lemma 2 (Section 4), defining $u^{i}, \lambda^{i}, g$, and $\varepsilon$ just as in that earlier proof, and obtaining the continuous, monotone, strictly concave function $U$, defined on $R^{n}$, as before.

Next, we apply the methods of Chiappori and Rochet (1987), pp. 690-691, to our $U$ as follows. Because $h$ is invertible and and the $\phi_{i}$ are continuous, each $x_{i}$ has a neighborhood $N\left(x_{i}, \delta_{i}\right)$ of radius $\delta_{i}>0$ such that:

$$
\forall x_{x \in N\left(x_{i}, \delta_{i}\right)} U(x)=\phi_{i}(x)
$$

Letting $\delta \equiv \min \left\{\delta_{i}: i=1, \ldots, k\right\}$, we define $V: R^{n} \rightarrow R$ by:

$$
V(x) \equiv \int_{R^{*}} U(x-\xi) \rho_{\delta}(\xi) d \xi
$$

where $\rho_{\delta}$ is the nonnegative, symmetric, $C^{\infty}$ function, defined in Chiappori and Rochet (1987), that vanishes outside the ball $N(0, \delta)$. In particular, define

$$
\rho(x) \equiv \begin{cases}\exp \left(-\frac{1}{\|x\|^{2}-1}\right) / \int_{R^{n}} \exp \left(-\frac{1}{\|x\|^{2}-1}\right) d x, & \text { if }\|x\|<1, \\ 0, & \text { otherwise, }\end{cases}
$$

and

$$
\rho_{\delta} \equiv \frac{1}{\delta} \rho\left(\frac{x}{\delta}\right) .
$$

Then, as a convolution, $V$ is $C^{\infty}$, and it is easily verified that $V$ is strictly concave and monotone on $R^{n}$, since $U$ had those properties.

Finally, we show that $V$ rationalizes $h$. First note that, for each $i=1, \ldots, k$,

$$
D V\left(x_{i}\right)=\lambda^{i} p^{i}
$$

For 


$$
\begin{aligned}
D V\left(x_{i}\right) & =\int_{N(0, \delta)} D \phi_{i}\left(x_{i}-\xi\right) \rho_{\delta}(\xi) d \xi \\
& =\int_{N(0, \delta)}\left(\lambda^{i} p^{i}-\varepsilon D g(-\xi)\right) \rho_{\delta}(\xi) d \xi \\
& =\lambda^{i} p^{i} \int_{N(0, \delta)} \rho_{\delta}(\xi) d \xi-\varepsilon \int_{N(0, \delta)} D g(-\xi) \rho_{\delta}(\xi) d \xi \\
& =\lambda^{i} p^{i}-0 .
\end{aligned}
$$

Now suppose $x \in X \& p^{i} x \leqq m^{i} \& x \neq x^{i}$. We must show that $V(x)<V\left(x^{i}\right)$. Since $V$ is strictly concave and differentiable, we have:

$$
\begin{aligned}
V(x) & <V\left(x^{i}\right)+D V\left(x^{i}\right)\left(x-x^{i}\right) \\
& =V\left(x^{i}\right)+\lambda^{i} p^{i}\left(x-x^{i}\right) \quad \text { by }(7.10) \\
& =V\left(x^{i}\right)+\lambda^{i}\left(p^{i} x-m^{i}\right) \\
& \leqq V\left(x^{i}\right) \quad \text { by hypothesis. }
\end{aligned}
$$

So $V$ rationalizes $h$. 


\section{REFERENCES}

AFRIAT, Sidney N. (1967): "The Construction of Utility Functions from Expenditure Data," International Economic Review, 8, 67-77.

CHIAPPORI, Pierre-André and Jean-Charles ROCHET. (1987): "Revealed Preferences and Differentiable Demand," Econometrica, 55, 687-691.

DIEWERT, W. E. (1973): "Afriat and Revealed Preference Theory," Review of Economic Studies, 40, 419-425.

GALE, David (1960): The Theory of Linear Economic Models. McGraw-Hill, New York, NY.

Guillemin, Victor and Alan POLlaCK (1974): Differential Topology. Prentice Hall, Englewood Cliffs, NJ.

HOUTHAKKER, Hendrik S. (1950): “Revealed Preference and the Utility Function," Economica, N.S., 17, 159-174.

KAHN, Donald W. (1980): Introduction to Global Analysis. Academic Press, New York, NY.

KIM, Taesung. (1987): “Intransitive Indifference and Revealed Preference," Econometrica, 55, 163-167.

MAS-COLELl, Andreu. (1978): "On Revealed Preference Analysis," Review of Economic Studies, 45, 121-131.

MATZKIN, Rosa L. (1986): “Mathematical and Statistical Inferences from Demand Data.,' Ph. D. dissertation, University of Minnesota, 1986.

RICHTER, Marcel K. (1966): “Revealed Preference Theory," Econometrica, 34, 635-645.

RICHTER, Marcel K. (1971): "Rational Choice," in Preferences, Utility, and Demand, John S. Chipman, Leonid Hurwicz, Marcel K. Richter and Hugo F. Sonnenschein, editors. Harcourt, Brace, Jovanovich, New York. Chapter 2.

RICHTER, Marcel K. (1979): “Duality and Rationality,' Journal of Economic Theory, 20, 131181. 
SAMUELSON, Paul A. (1938): "A Note on the Pure Theory of Consumer's Behaviour," Economica, N.S., 5, 61-71.

SAmUelson, Paul A. (1938): "A Note on the Pure Theory of Consumer's Behaviour: An Addendum," Economica, N.S., 5, 353-354.

STOER, Josef and Christoph WITZGALL (1970): Convexity and Optimization in Finite Dimensions I. Springer-Verlag, New York.

UZAWA, Hirofumi, (1971): "Preference and Rational Choice in the Theory of Consumption," in Preferences, Utility, and Demand, John S. Chipman, Leonid Hurwicz, Marcel K. Richter and Hugo F. Sonnenschein, editors. Harcourt Brace Jovanovich, New York. Chapter 1.

VARIAN, Hal R. (1982): “The Nonparametric Approach to Demand Analysis," Econometrica, 50, 945-973. 